\title{
激发态分子内质子转移(ESIPT)发色团修饰的树枝形聚合物合成及 光物理研究
}

\author{
李鹏曾毅*陈金平李迎迎李嫕* \\ (光化学转换与功能材料重点实验室 中国科学院理化技术研究所 北京 100190)
}

\begin{abstract}
摘要 设计合成了 $0 \sim 4$ 代外围修饰激发态分子内质子转移(ESIPT)发色团的聚酰胺-胺树枝形聚合物 G0 $\mathrm{G} 4$, 化合物 结构经过 IR, ${ }^{1} \mathrm{H}$ NMR, ${ }^{13} \mathrm{C}$ NMR 和 MS 表征. 稳态光谱研究表明, 树枝形聚合物在四氢呋喃溶液中形成了聚集体, 发色 团酮式发光随着化合物代数增大呈先增加后减小的变化. 质子化树枝形聚合物 $\mathrm{G} 1-\mathrm{H} \sim \mathrm{G} 4-\mathrm{H}$ 能溶于水, 并在水中形成 $20 \mathrm{~nm}$ 左右的聚集体, 发色团在聚集体疏水区中构象受限, 仅发射酮式发光, 并且发光强度受树枝形聚合物分子大小的 影响.
\end{abstract}

关键词＼cjkstart聚酰胺-胺树枝形聚合物; 激发态分子内质子转移; 光物理

\section{Synthesis and Photophysical Study of Dendrimers Modified with ESIPT Chromophore}

\author{
Li, Peng \\ Zeng, $\mathrm{Yi}^{*}$ \\ Chen, Jinping \\ Li, Yingying \\ Li, Yi* \\ (Key Laboratory of Photochemical Conversion and Optoelectronic Materials, Technical Institute of Physics and Chemistry,
} Chinese Academy of Sciences, Beijing 100190)

\begin{abstract}
A series of excited-state intramolecular proton transfer (ESIPT) poly(amido amine) (PAMAM) dendrimers with 4-(benzo[ $d]$ thiazol-2-yl)-3-hydroxyphenyl modified at the periphery were synthesized (G0-G4). Functionalization of PAMAM dendrimers was performed through the condensation reaction of dendrimer peripheral amine units and carboxylic groups in DMSO catalyzed by 1-ethyl-3-(3-dimethyllaminopropyl) carbodiimide hydrochloride (EDC). The structures of synthetic compounds G0- G4 were characterized by IR, ${ }^{1} \mathrm{H}$ NMR, ${ }^{13} \mathrm{C}$ NMR, and MS. The average functionalization extents of the peripheral amine groups were $99 \%, 88 \%, 80 \%, 83 \%$, and $78 \%$ for G0-G4, respectively. The solubility of functionalized dendrimers is dramatically affected by peripheral groups and the dendritic backbone. Model compound dissolves in THF homogeneously, exhibiting enol emission only due to the freely intramolecular rotation and consequently few ESIPT process occurrence. On the contrary, dendrimers form aggregates in THF and present keto emission of ESIPT chromophores, which increases with the dendrimer generation increasing from 0 to 2 and decreases in generation 3 and 4, indicating that the emission of ESIPT chromophores is affected by the PAMAM backbone and peripheral units. From G0 to G2, the increasing congestion of aggregates restrains the intramolecular rotation of ESIPT chromophores, which facilitates the ESIPT process and results in more keto emission. However, a considerable enol emission presents in G3 and G4 because larger dendritic backbone provides more free space and higher polarity microenvironment. The protonated dendrimers, G1-H-G4-H, are soluble in water and form aggregates with a diameter of $c a .20 \mathrm{~nm}$ demonstrated by dynamic light scattering experiments. TEM image of aggregates was also obtained revealing vesicle like assemblies. The absorption of ESIPT groups is not affected by protonation of dendritic backbones giving typical absorption of the ESIPT chromophore. The ESIPT chromophore exhibits keto emission exclusively due to the restricted conformation by confined aggregation microenvironments in water, and the emission intensity is slightly affected by dendritic backbones. The lower emission intensity of G4-H can be attributed to the loose microenvironment in the G4-H assembly caused by the larger and flexible dendrimer backbone.

Keywords PAMAM dendrimer; excited-state intramolecular proton transfer (ESIPT); photophysics
\end{abstract}

\section{1 引言}

树枝形聚合物是一类围绕中心核，外围链段随着代 数的增加以指数级别增长的支化大分子, 与传统高分子
相比它具有高度的几何对称性，精确的分子结构，分子 内存在空腔, 分子大小为纳米尺寸等特点 ${ }^{[1,2]}$. 在树枝形 聚合物的核心、骨架和外围修饰上各种功能基团可以实 现树枝形聚合物的功能化，树枝形聚合物在超分子组

\footnotetext{
*E-mail: yili@mail.ipc.ac.cn; zengyi@mail.ipc.ac.cn

Received March 19, 2012; published April 17, 2012.

Supporting information for this article is available free of charge via the Internet at http://sioc-journal.cn.

Project supported by the National Natural Science Foundation of China (Nos. 21004072, 21002109, 21073215, 21173245) and the National Basic Research Program (No. 2010CB934500).

项目受国家自然科学基金(Nos. 21004072, 21002109, 21073215, 21173245)和科技部国家重点基础研究发展计划(No. 2010CB934500)资助.
} 
装 $^{[3,4]}$, 生物医药 ${ }^{[5]}$, 化学传感器 ${ }^{[6]}$, 微反应器 ${ }^{[7,8]}$, 光电 材料 ${ }^{[9,10]}$ 以及模拟光捕获体系 ${ }^{[11]}$ 等研究领域已得到广泛 应用。

激发态分子内质子转移(Excited-State Intramolecular Proton Transfer, ESIPT)化合物具有特殊的激发态光 物理过程, 即 ESIPT 化合物在激发态分子内某一基团上 的氢核(即质子)能够通过分子内氢键转移到邻近的 $\mathrm{N}$, $\mathrm{S}, \mathrm{O}$ 等杂原子上, 形成互变异构体, 典型的 ESIPT 化合 物有 $\beta$-差基丙稀醛、邻羟基苯甲醛、水杨酸、7-羟基茆 酮、羟基黄酮类以及 2-(2'-羟基苯基)苯并唑类等 ${ }^{[12]}$. 通 过基态和激发态间烯醇式(Enol)-酮式(Keto)转换, ESIPT 化合物具有独特的 $E-E^{*}-K^{*}-K-E$ 四能级跃迁行为, 使这 类化合物的吸收和发射光谱之间具有很大的斯托克斯 位移(Stokes's shift). 这种大的斯托克斯位移消除了发 光分子由于自吸收导致的发光猝灭, 增大了在聚集态或 固态下得到强发光有机材料的可能性. 基于 ESIPT 的独 特性质, 研究人员在理论和实验上对这类化合物开展了 大量研究, 并将其应用到化学传感器、有机发光材料、 激光染料等领域 ${ }^{[12 ~ 19]}$.

将 ESIPT 发色团修饰到树枝形聚合物外围可以实 现分子光功能化, 树枝形聚合物的特殊结构和构象以及 官能团间的相互作用将对化合物的光物理性质有很大 的影响, 使其具有不同于传统分子的性质. 设计合成 ESIPT 基团修饰的树枝形聚合物, 了解功能化树枝形聚 合物结构与性质之间的关系, 对发展有机光捕获体系和 有机光功能材料有重要意义. 本工作设计合成了一系列 外围修饰2-(2'-差基苯基)苯并噻唑(HBT)的单支聚酰胺胺树枝形聚合物(PAMAM), 用 IR, ${ }^{1} \mathrm{H}$ NMR, ${ }^{13} \mathrm{C} \mathrm{NMR}$ 和 MS 对其结构进行了表征, 通过吸收和荧光光谱研究 了化合物在四氢呋喃溶液中的光物理性质, 以及质子化 后化合物在水中的光谱性质.

\section{2 结果与讨论}

\section{1 树枝形聚合物的合成及修饰}

以炔丙胺为起始核心原料合成单支 PAMAM 树枝 形聚合物骨架，外围分别含有 2 32 个氨基可供衍生化 修饰. 树枝形聚合物外围经过官能化修饰后, 核心的炔 基可以方便地通过 Click Chemistry 或 Sonogashira Reaction 修饰上核心基团或将树枝形聚合物修饰到特定基 底上, 实现光捕获或化学传感等功能. 本文仅对树枝形 聚合物的外围官能化过程以及四氢呋喃和水溶液中的 稳态光物理性质进行讨论.

本工作所合成的 ESIPT 发色团含有苯胺基, 但该氨 基亲和能力弱, 不能通过氨解反应直接将发色团修饰到 PAMAM 的末端. 因此, 我们首先通过苯胺基团与丁二 酸酐进行酰化反应, 得到含一个游离羧酸的酰胺中间产 物, PAMAM 末端氨基与酰胺中间产物的游离羧基在 EDC 的作用下进行缩合反应, 得到 ESIPT 基团修饰的树
枝形聚合物. 这种衍生化途径所涉及的反应条件温和, 而且还避免了合成过程中苯环羟基基团的保护与去保 护过程，简化了反应的步骤，减少了提纯处理过程中的 损失. 根据核磁共振氢谱计算得到 $\mathrm{G} 0 \sim \mathrm{G} 4$ 外围氨基官 能化的比例分别为: $99 \%, 88 \%, 80 \%, 83 \%$ 和 $78 \%$. 外围 修饰 ESIPT 发色团的树枝形聚合物不溶于水, 在常见有 机溶剂如甲醇、乙醇、丙酮、乙酸乙酯、氯仿、二氯甲 烷等中溶解度较差, 微溶于四氢呋喃. 将 PAMAM 骨架 质子化后, ESIPT 发色团修饰的树枝形聚合物在水中的 溶解度大大提高. 由此可见, 外围修饰的官能团和 PAMAM 骨架对树枝形聚合物的溶解性均有很大影响. 图 1 给出了 Model 和各代官能化树枝形聚合物的核磁共 振氢谱.

\section{2 化合物在 THF 中的光谱性质}

图 $2 \mathrm{a}$ 给出了在相同官能团浓度下 Model 和 $\mathrm{G} 0 \sim \mathrm{G} 4$ 树枝形聚合物在 THF 中的吸收光谱. Model 的吸收光谱 是典型的羟苯基苯并噻唑的吸收, 在 296, 308, 348 和 $363 \mathrm{~nm}$ 处有明显的吸收峰, 而且吸收峰的结构清晰. $\mathrm{G} 0 \sim \mathrm{G} 4$ 树枝形聚合物的吸收光谱形状与 Model 相类似， 但吸光度大小和吸收峰的精细结构发生了明显变化. 随 着树枝形聚合物代数增加, $350 \mathrm{~nm}$ 附近吸收峰的吸光度 呈现先下降后增加的变化, 而且吸收峰逐渐失去精细结 构, 其中 $\mathrm{G} 2$ 的吸收最低, 吸收峰结构最不清晰, 此外树 枝形聚合物吸收光谱长波长区域出现散射导致的拖尾 现象，并且树枝形聚合物的体系可以观察到丁达尔现 象, 由此推测各代数树枝形聚合物在四氢呋喃溶液中均 形成了聚集体，聚集体的形成使树枝形聚合物的吸收光 谱与 Model 相比发生较大变化.

以 $350 \mathrm{~nm}$ 的光激发 Model 和 $\mathrm{G} 0 \sim \mathrm{G} 4$ 树枝形聚合 物, 得到图 $2 \mathrm{~b}$ 所示的苂光光谱, 化合物均呈现两组发射 峰: $385 \mathrm{~nm}$ 附近的发射峰来自 ESIPT 发色团的醇式激发 态, $516 \mathrm{~nm}$ 处的发光则归属于激发态分子内质子转移所 形成的酮式激发态的发射. Model 基本上以醇式发光为 主，长波长区域的酮式发光非常微弱，表明模型化合物 在溶液中发生 ESIPT 的比例很少. 根据 ESIPT 化合物发 光机理可以得知, 发色团的双菼光发射性质对微环境极 性和聚集态下的构象分布十分敏感, 弱的极性环境以及 平面化的构象状态利于发生 ESIPT 过程, 得到酮式发 光 $^{[12]}$. 模型化合物在四氢呋喃中处于单分散状态, 着茎 基和苯并㗌唑基均可以绕连接的单键自由旋转, 不利于 发生激发态分子内质子转移, 因此 Model 主要表现为醇 式发光. 树枝形聚合物的发光随着代数的不同存在明显 差异，其中酮式发光强度随化合物代数增大呈先增加后 下降的趋势. 酮式发光的显现是由于树枝形聚合物在四 氢呋喃中形成聚集体, 根据 $\mathrm{G} 0 \sim \mathrm{G} 4$ 的吸收光谱和荧光 光谱随代数的变化(图 2c) 推测, 随着 $\mathrm{G} 0 \sim \mathrm{G} 2$ 树枝形聚 合物代数增加，化合物在四氢呋喃中更容易形成聚集 体，发色团构象旋转受限程度增大，其结构更趋近于平 
面化, 有利于激发态分子内质子转移过程的发生, 因而 酮式发光随代数增加而增强. 当树枝形聚合物代数继续 增加, 虽然高代数化合物 G3 和 G4 更易形成聚集体, 但 由于化合物分子结构中柔性 PAMAM 骨架比例较高, 骨 架内空腔也较多, 发色团在聚集体中构象旋转受限相对
减弱，使得发色团平面化构象减少，而且聚酰胺一胺骨 架的极性也较高，减少了激发态分子内质子转移过程, 酮式发光也随之减弱. 不同聚集体中发色团的构象分布 特点以及发光特性可以用图式 2 表示.
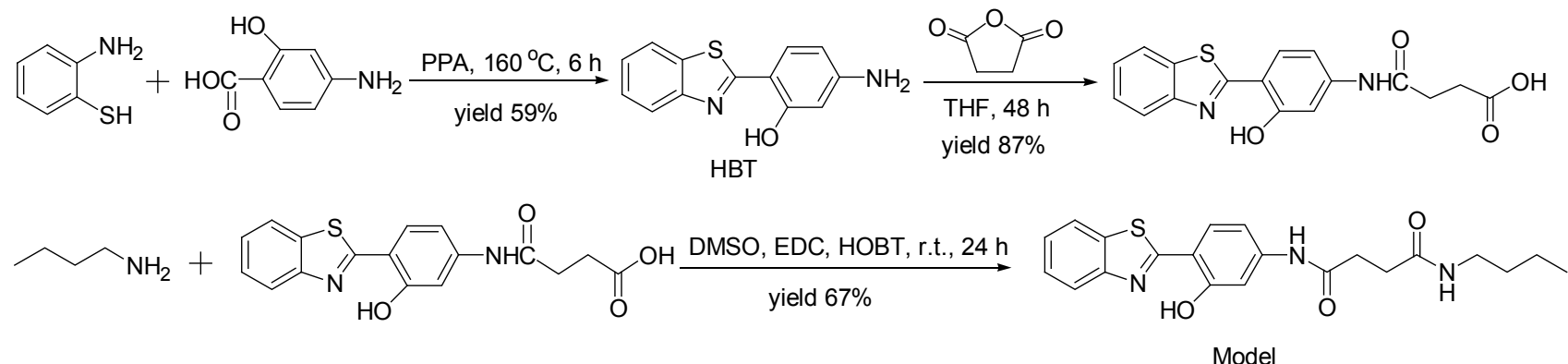<smiles>C#CCN(CCNC(N)CN)CCC(=O)NCCN</smiles>

gn

$n=0,1,2,3,4$<smiles>O=CCCC(=O)Nc1ccc(-c2nc3ccccc3s2)c(O)c1</smiles>

DMSO, EDC, HOBT, r.t., 4 days yield $57 \% \sim 67 \%$

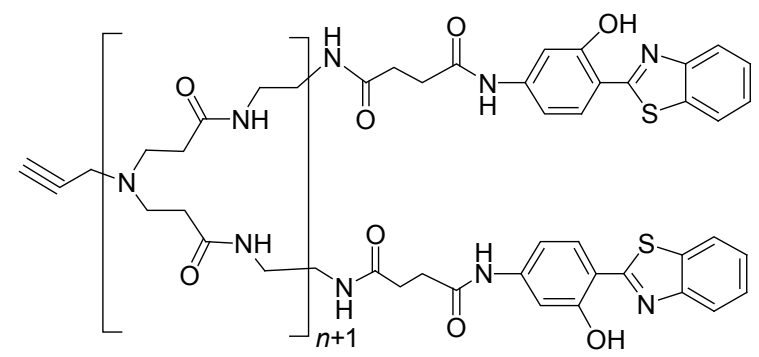

$\mathrm{Gn}$

$n=0,1,2,3,4$

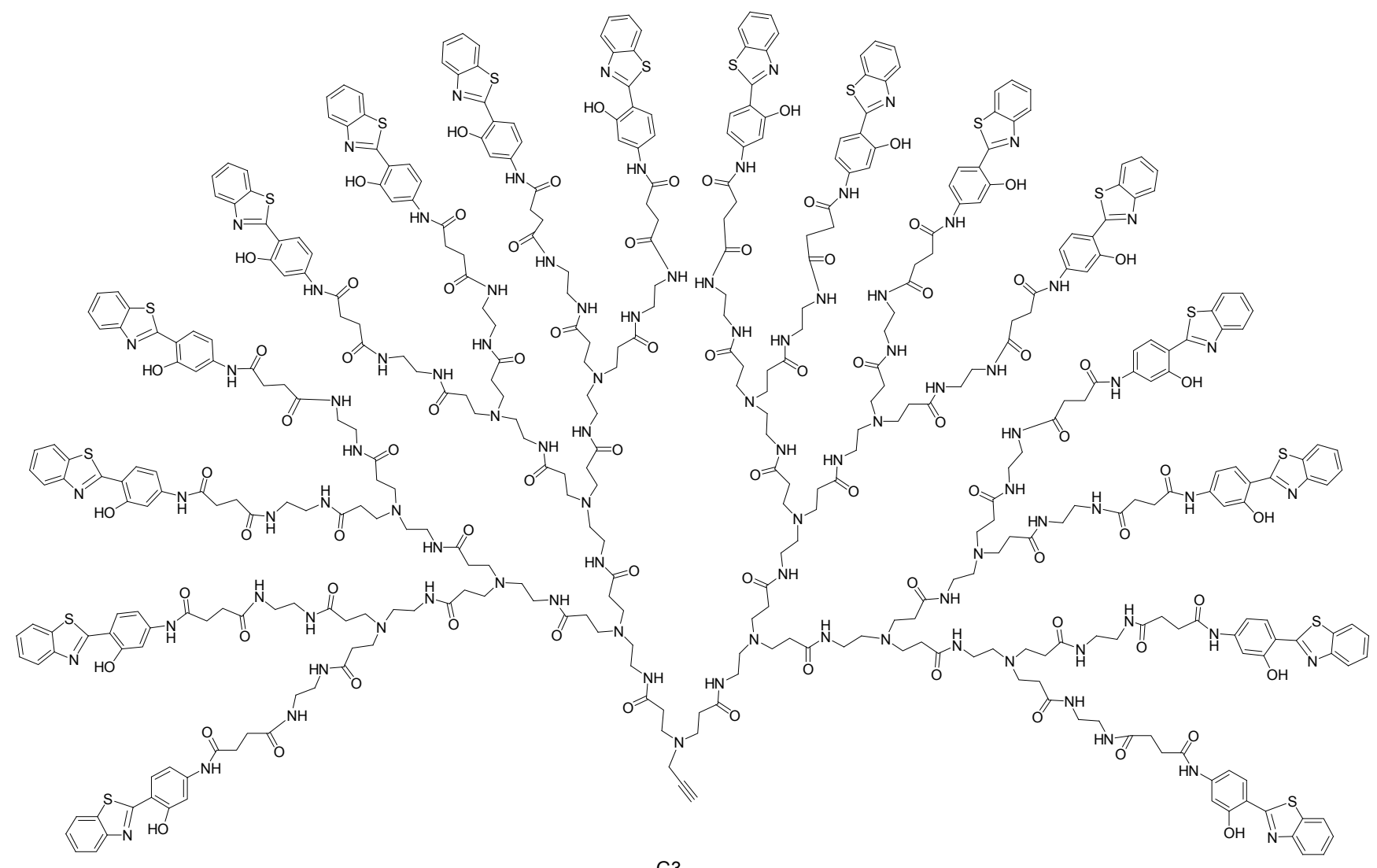

G3

图式 1 化合物合成路线和 G3 树枝形聚合物结构式

Scheme 1 Synthetic route of compounds and structure of G3 dendrimer 


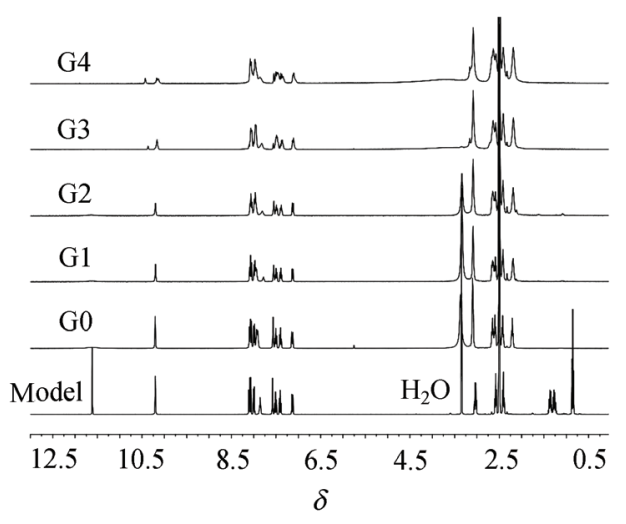

图 1 Model 和 $\mathrm{G} n$ 的 ${ }^{1} \mathrm{H}$ NMR 图(400 MHz, DMSO- $d_{6}$ )

Figure $1{ }^{1} \mathrm{H}$ NMR spectra of Model and G $n\left(400 \mathrm{MHz}, \mathrm{DMSO}-d_{6}\right)$

\section{3 质子化树枝形聚合物 $\mathrm{G} n-\mathrm{H}$ 在水中聚集态及光物 理研究}

PAMAM 骨架具有良好的水溶性, 但 $\mathrm{G} 0 \sim \mathrm{G} 4$ 由于 修饰有较大的疏水性 ESIPT 发色团, 均不溶于水, 将 PAMAM 骨架质子化能够大大增加树枝形聚合物在水 中的溶解度 ${ }^{[4]}$. 我们将 $0 \sim 4$ 代树枝形聚合物用盐酸进行 质子化后得到 $\mathrm{G} n-\mathrm{H}$, 除了 $\mathrm{G} 0-\mathrm{H}$ 因树枝形聚合物骨架结 构太小在水中的溶解度仍然很低外, 其余各代树枝形聚 合物 $\mathrm{G} 1-\mathrm{H} \sim \mathrm{G} 4-\mathrm{H}$ 均能溶于水. $\mathrm{G} 1-\mathrm{H} \sim \mathrm{G} 4-\mathrm{H}$ 在水中的 吸收光谱如图 3a 所示, 各代树枝形聚合物的吸收均表 现为 ESIPT 发色团的特征吸收, 吸收峰在 296, 336 和 $367 \mathrm{~nm}$, 化合物的摩尔消光系数随着化合物代数的增加 成比例增加, 表明质子化对化合物的吸收没有明显影 响. 用 $350 \mathrm{~nm}$ 的光激发树枝形聚合物水溶液测得图 $3 \mathrm{~b}$ 所示的荧光光谱图, 质子化的树枝形聚合物在水中均只 呈现酮式发光, 表明水溶液中发色团极易发生 ESIPT 过 程. 动态光散射实验(DLS)研究发现, 1 4 代树枝形聚 合物在水中均形成了直径约 $20 \mathrm{~nm}$ 的聚集体, 以 $\mathrm{G} 3-\mathrm{H}$ 为例, 通过 TEM 研究了形成聚集体的形貌, 如图 3c 所 示. 树枝形聚合物含有亲水的质子化 PAMAM 骨架和疏 水的 ESIPT 发色团, 是两亲性结构, 并且具有柔性骨架, 根据文献 ${ }^{[20,21]}$ 可以推断, 不同代数的树枝形聚合物树枝 形聚合物在水中可以形成尺寸相近的囊泡状聚集体, 疏 水的发色团在聚集体疏水区域紧密堆积, 处于有利于发 生 ESIPT 的构象. 从归一化的荧光光谱可以看出, G1-H 和 G2-H 的荧光强度基本相同, G3-H 略强, G4-H 最低. 根据文献报道 ${ }^{[12,22]}$, 构象旋转受限不仅是 ESIPT 化合物 产生酮式发光的原因, 也是其聚集荧光增强的原因, 因 此, 我们推断不同代数树枝形聚合物水溶液荧光强度的 差异来源于 ESIPT 基团所受限制作用不同. 各代树枝形 聚合物的 PAMAM 骨架大小和发色团数量不同导致形 成聚集体的限制性微环境不同, 1 代和 2 代骨架相对较 小, 发色团数量较少, 形成的聚集体对构象旋转的限制 作用相近, 发色团的发光强度也基本一致; 3 代化合物 聚集形成的聚集体结构相比 1 2 代较为紧密, 发色团
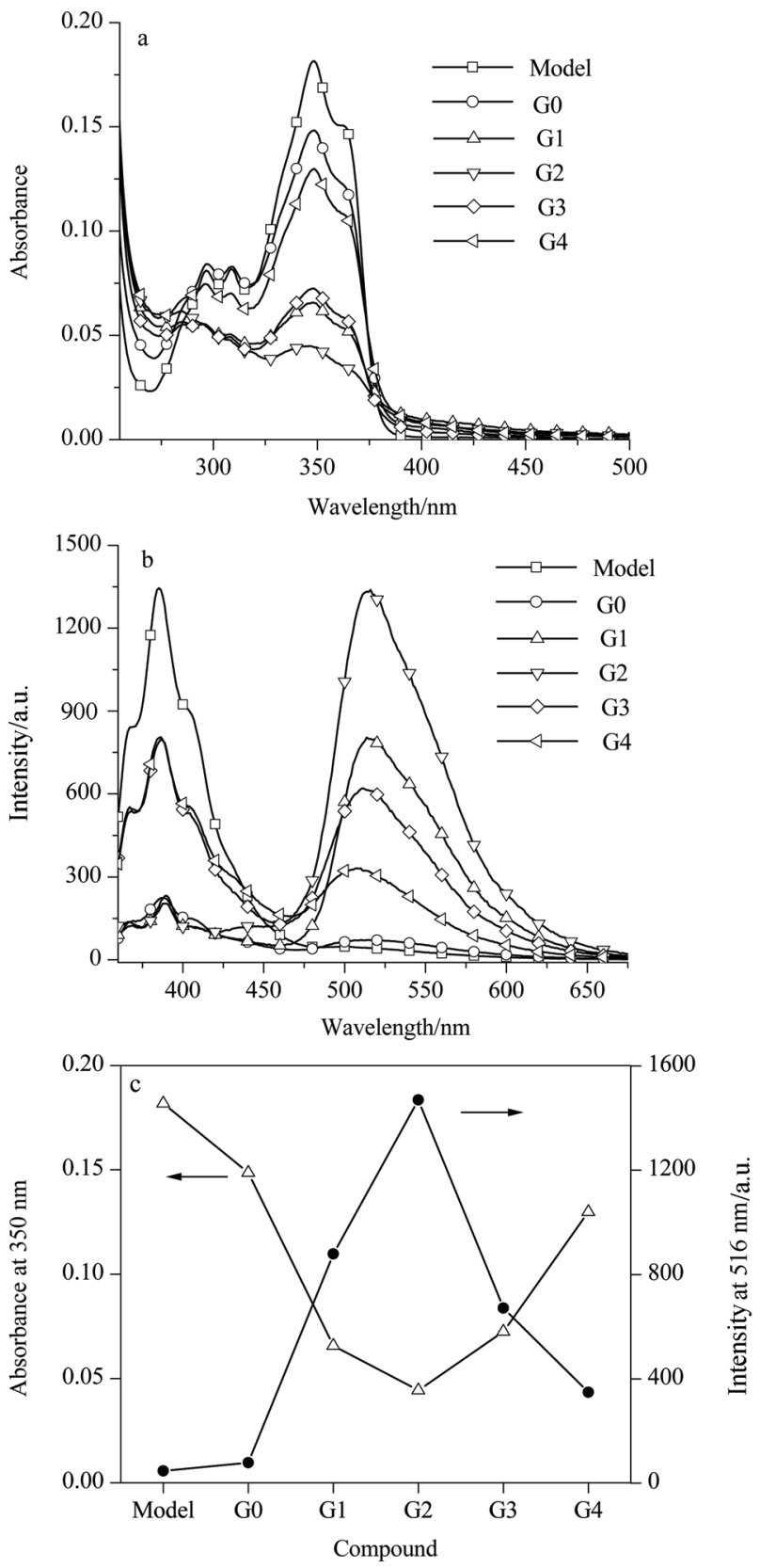

图 2 Model 及 $\mathrm{G} n$ 在 THF 中的吸收光谱(a)和对激发波长处吸收归一 化的苂光光谱(b), 各化合物在 $350 \mathrm{~nm}$ 处吸光度和 $516 \mathrm{~nm}$ 处苂光强度 的变化趋势图 (c), 发色团浓度为 $5 \times 10^{-6} \mathrm{~mol} \cdot \mathrm{L}^{-1}, \lambda_{\mathrm{ex}}=350 \mathrm{~nm}$.

Figure 2 Absorption spectra of Model and $\mathrm{G} n$ (a) and emission spectra of Model and $\mathrm{G} n$ normalized to the absorbance at the excitation wavelength (b) in THF. Changes of absorbance at $350 \mathrm{~nm}$ and emission intensity at $516 \mathrm{~nm}$ of the compounds (c), $[\mathrm{HBT}]=5 \times 10^{-6} \mathrm{~mol} \cdot \mathrm{L}^{-1}, \lambda_{\text {ex }}=350$ $\mathrm{nm}$

在聚集体中受到的构象限制稍大，更多地抑制了非辐射 失活, 发光比 1,2 代化合物略强; 4 代化合物虽然发色团 数量和树枝形聚合物骨架体积相比低代数化合物都大 大增加，但其发光强度最弱，推测是较大 4 代树枝形聚 合物分子形成的聚集体结构较为松散, 位于其中的发色 团的构象受限较弱, 减少了聚集导致的苂光强度增加幅 度. 


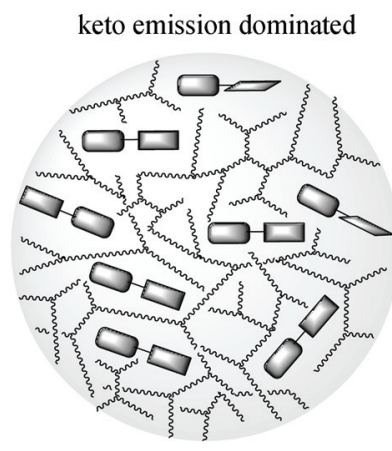

Gn aggregate $(n=0 \sim 2)$

mav $^{m^{w}}=$ dendrimer backbone

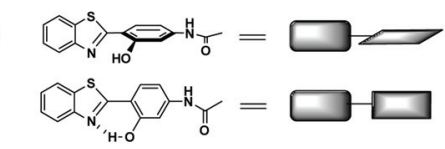

图式 2 不同代数树枝形聚合物形成聚集体中发色团的构象及发光特 征示意图

Scheme 2 Visual expression of the conformation and the emission of chromophores varied with the aggregate forming from different generation dendrimers.

\section{3 结论}

合成了一系列 ESIPT 发色团修饰的树枝形聚合物 $\mathrm{G} 0 \sim \mathrm{G} 4$, 研究了化合物在四氢呋喃中的光物理性质, 探讨了 PAMAM 骨架质子化对化合物在水中溶解度的 影响以及对发光性质的影响. 研究结果表明, ESIPT 发 色团修饰的树枝形聚合物在四氢呋喃中以聚集体形式 存在, 由于发色团和骨架的共同作用使得发色团在聚集 体中的状态随代数变化, 发色团的酮式发光随代数增大 呈现先增加后减小的变化. 树枝形聚合物质子化后亲水 性得到很大改善, 质子化对化合物的吸收没有明显影 响, 质子化树枝形聚合物在水中形成直径约 $20 \mathrm{~nm}$ 的聚 集体, 发色团在聚集体中易发生 ESIPT 过程, 仅呈现酮 式发光, 不同代数树枝形聚合物形成的聚集体中构象限 制微环境不同，导致不同代数化合物的荧光强度产生差 异.

\section{4 实验部分}

ESIPT 发色团[2-(2'-羟基-4-氨基苯基)苯并噻唑 (HBT) ]的合成 ${ }^{[15]}$ : 产率 $59 \%$.

$N$-[3-羊基-4-(2'-苯并噻唑)苯基]丁二酸单酰胺的合 成: 在 $100 \mathrm{~mL}$ 三口瓶中加入 $15 \mathrm{mmol}$ 丁二酸酐和 $40 \mathrm{~mL}$ THF, 待丁二酸酐溶解后加入 $1 \mathrm{mmol} 2$-(2'-差基-4-氨基 苯基)苯并噻唑, 摚拌回流反应 $48 \mathrm{~h}$. 反应物冷却至室温 后过滤, 依次用 THF、二氯甲烷、 $1 \mathrm{~mol} / \mathrm{L}$ 盐酸和水洗 涤固体, 将固体放在真空烘箱中于 $60{ }^{\circ} \mathrm{C}$ 下干燥, 得到 产物，产率 $87 \%$.

模型化合物(Model)的合成: 在 $50 \mathrm{~mL}$ 两口瓶中加 入 $3 \mathrm{mmol} N$-[3-羊基-4-(2'-苯并噻唑)苯基]丁二酸单酰胺 和 $15 \mathrm{~mL}$ DMSO, 待固体溶解后依次加入 $3.6 \mathrm{mmol}$
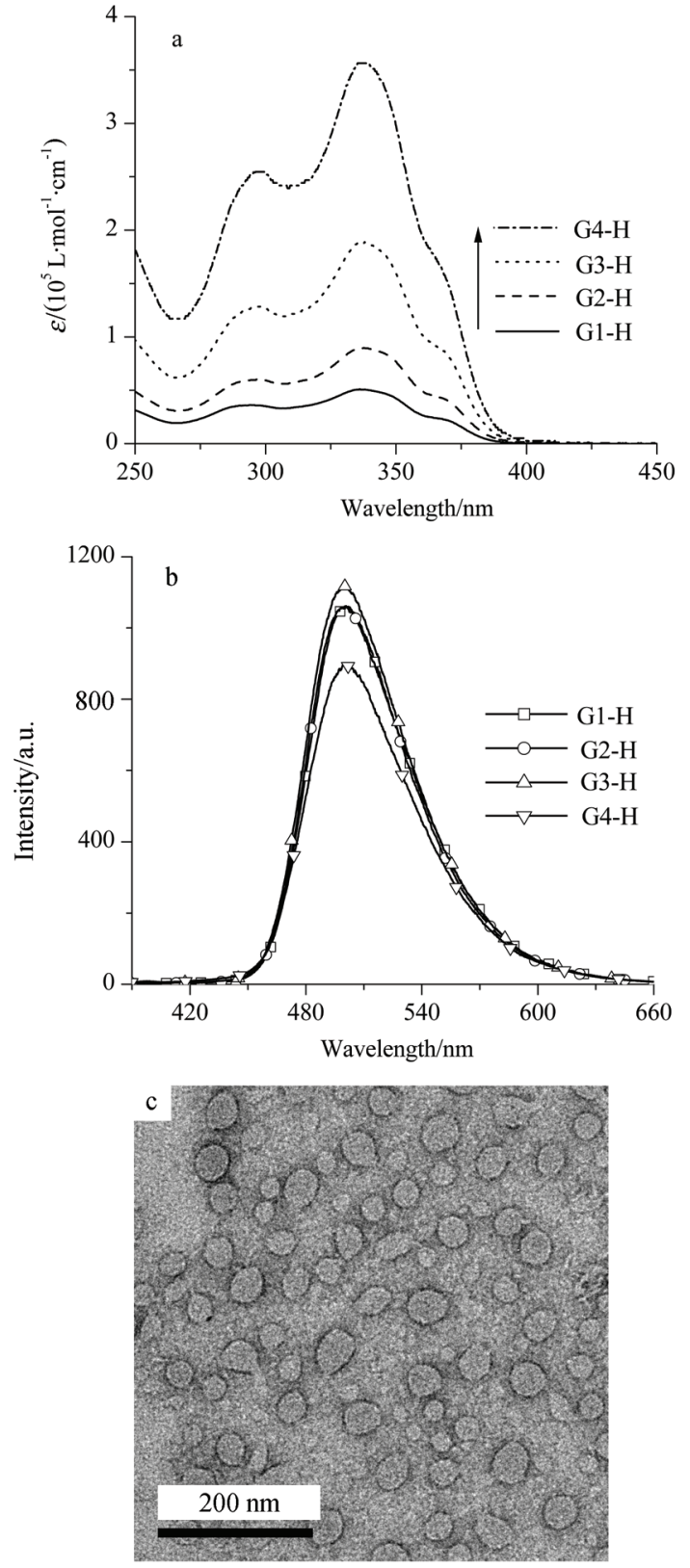

图 3 质子化树枝形聚合物 $\mathrm{G} 1-\mathrm{H} \sim \mathrm{G} 4-\mathrm{H}$ 在水中的吸收光谱(a)和对激 发波长处吸收归一化的荧光光谱(b), 发色团浓度为 $5 \times 10^{-6} \mathrm{~mol} \cdot \mathrm{L}^{-1}$, $\lambda_{\mathrm{ex}}=350 \mathrm{~nm}$. G3-H 在水中形成聚集体的 TEM 图(c).

Figure 3 (a) Absorption spectra of G1-H-G4-H; (b) emission spectra of $\mathrm{G} 1-\mathrm{H}-\mathrm{G} 4-\mathrm{H}$ normalized to the absorbance at the excitation wavelength in water, $[\mathrm{HBT}]=5 \times 10^{-6} \mathrm{~mol} \cdot \mathrm{L}^{-1}, \lambda_{\mathrm{ex}}=350 \mathrm{~nm}$; and (c) TEM image of aggregates formed by $\mathrm{G} 3-\mathrm{H}$ in aqueous solution.

1-(3-二甲氨基丙基)-3-乙基碳二亚胺盐酸盐, $3 \mathrm{mmol}$ 1羟基苯并三氮唑(HOBT). 氮气保护下室温摚拌反应 $2 \mathrm{~h}$, 向反应体系缓慢滴加 $15 \mathrm{~mL}$ 溶有 $3.6 \mathrm{mmol}$ 正丁胺的 DMSO 溶液, 继续搅拌反应 $24 \mathrm{~h}$, 将反应液慢慢倒入稀 碳酸氢钠溶液中, 过滤并用适量水洗涤固体, 用少量 DMSO 溶解固体，在水中沉淀一次，最后通过 THF/DMSO $(V / V=25: 1)$ 重结晶得到 Model, 产率 $67 \%$. 
PAMAM 树枝形聚合物骨架根据文献报道的发散 法合成 ${ }^{[23]}$. 以炔丙胺为核心出发, 与丙烯酸甲酯进行 Michael 加成反应, 待氨基反应完全后减压除去未反应 的丙烯酸甲酯，得到末端为甲酯的树枝形分子，然后在 氮气保护下用过量的乙二胺进行氨解, 减压蒸馏除去过 量的反应物后得到 0 代 PAMAM, 重复这两步反应得到 $0 \sim 4$ 代单支 PAMAM 树枝形聚合物骨架 $(\mathrm{g} n, n=0 \sim 4)$.

PAMAM 末端胺基修饰过程如图式 1 所示, 以 4 代 树枝形聚合物为例: 在 $50 \mathrm{~mL}$ 两口瓶中加入 $3 \mathrm{mmol}$ $N$-[3-美基-4-(2'-苯并噻唑)苯基]丁二酸单酰胺和 $15 \mathrm{~mL}$ DMSO, 待固体溶解后依次加入 $3.6 \mathrm{mmol} 1$-(3-二甲氨 基丙基)-3-乙基碳二亚胺盐酸盐, $3 \mathrm{mmol}$ 1-羟基苯并三 氮唑(HOBT), 氮气保护下室温搅拌反应 $2 \mathrm{~h}$, 向反应体 系缓慢滴加 $20 \mathrm{~mL}$ 溶有 $0.06 \mathrm{mmol} \mathrm{g} 4$ 的 DMSO 溶液, 苯 并噻唑发色团与 $\mathrm{g} 4$ 外围胺基的物质的量之比为 $1.4: 1$, 继续摚拌反应 $96 \mathrm{~h}$, 将反应液慢慢倒入稀碳酸氢钠溶液 中, 过滤并用适量水洗涤固体, 用少量 DMSO 溶解固 体, 在水中沉淀一次, 收集的固体再在 $\mathrm{CH}_{2} \mathrm{Cl}_{2} / \mathrm{CH}_{3} \mathrm{OH}$ $(V / V=10: 1)$ 混合溶剂中沉淀三次, 过滤并用适量 $\mathrm{CH}_{3} \mathrm{OH}$ 洗涤, 在真空烘箱中于 $60{ }^{\circ} \mathrm{C}$ 下干燥, 得到浅黄 色固体产物 $\mathrm{G} 4$, 产率为 $57 \%$. 利用同样的方法合成得到 $\mathrm{G} 0 \sim \mathrm{G} 3$ 树枝形聚合物, 产率分别为 $65 \%, 67 \%, 60 \%$, $61 \%$, 均为浅黄色固体. 化合物结构详细表征数据见 Supporting Information.

\section{References}

[1] Bosman, A. W.; Janssen, H. M.; Meijer, E. W. Chem. Rev. 1999, 99, 1665.

[2] Grayson, S. M.; Frechet, J. M. J. Chem. Rev. 2001, 101, 3819.
[3] Smith, D. K.; Hirst, A. R.; Love, C. S.; Hardy, J. G.; Brignell, S. V.; Huang, B. Q. Prog. Polym. Sci. 2005, 30, 220.

[4] Zeng, Y.; Li, Y. Y.; Li, M.; Yang, G. Q.; Li, Y. J. Am. Chem. Soc. 2009, 131,9100

[5] Tekade, R. K.; Kumar, P. V.; Jain, N. K. Chem. Rev. 2009, 109, 49.

[6] Kikkeri, R.; Garcia-Rubio, I.; Seeberger, P. H. Chem. Commun. 2009, 235.

[7] Yuan, Z.; Chen, J. P.; Zeng, Y.; Li, Y. Y.; Han, Y. B.; Li, Y. Org. Biomol. Chem. 2011, 9, 6256.

[8] Yuan, Z.; Zheng, S. J.; Zeng, Y.; Chen, J. P.; Han, Y. B.; Li, Y. Y.; Li, Y. New J. Chem. 2010, 34, 718.

[9] Zou, J. H.; Wu, H.; Lam, C. S.; Wang, C. D.; Zhu, J.; Zhong, C. M.; Hu, S. J.; Ho, C. L.; Zhou, G. J.; Wu, H. B.; Choy, W. C. H.; Peng, J. B.; Cao, Y.; Wong, W. Y. Adv. Mater. 2011, 23, 2976.

[10] Lo, S. C.; Burn, P. L. Chem. Rev. 2007, 107, 1097.

[11] Zeng, Y.; Li, Y. Y.; Chen, J. P.; Yang, G. Q.; Li, Y. Chem. Asian J. 2010, $5,992$.

[12] Kwon, J. E.; Park, S. Y. Adv. Mater. 2011, 23, 3615.

[13] Li, S. Y.; He, L. M.; Xiong, F.; Li, Y.; Yang, G. Q. J. Phys. Chem. B 2004, 108, 10887.

[14] Lv, F. T.; Gao, L. N.; Fang, Y. Prog. Chem. 2005, 17, 773. (吕风婷, 高莉宁, 房喻, 化学进展, 2005, 17, 773.)

[15] Qian, Y.; Li, S. Y.; Zhang, G. Q.; Wang, Q.; Wang, S. Q.; Xu, H. J.; Li, C. Z.; Li, Y.; Yang, G. Q. J. Phys. Chem. B 2007, 111, 5861.

[16] Park, S.; Kwon, J. E.; Kim, S. H.; Seo, J.; Chung, K.; Park, S.-Y.; Jang, D.-J.; Medina, B. M.; Gierschner, J.; Park, S. Y. J. Am. Chem. Soc. 2009, 131, 14043.

[17] Hu, R.; Feng, J. A.; Hu, D. H.; Wang, S. Q.; Li, S. Y.; Li, Y.; Yang, G. Q. Angew. Chem., Int. Ed. 2010, 49, 4915.

[18] Qian, Y.; Cai, M. M.; Xie, L. H.; Huang, W. Acta Chim. Sinica 2010, 68, 2285. (钱妍, 蔡敏敏, 解令海, 黄维, 化学学报, 2010, $68,2285$.

[19] Tang, K. C.; Chang, M. J.; Lin, T. Y.; Pan, H. A.; Fang, T. C.; Chen, K. Y.; Hung, W. Y.; Hsu, Y. H.; Chou, P. T. J. Am. Chem. Soc. 2011, 133, 17738 .

[20] Schenning, A. P. H. J.; Elissen-Roman, C.; Weener, J. W.; Baars, M. W. P. L.; van der Gaast, S. J.; Meijer, E. W. J. Am. Chem. Soc. 1998, $120,8199$.

[21] Wang, B. B.; Zhang, X.; Jia, X. R.; Li, Z. C.; Ji, Y.; Yang, L.; Wei, Y. J. Am. Chem. Soc. 2004, 126, 15180.

[22] Hu, R.; Li, S. Y.; Zeng, Y.; Chen, J. P.; Wang, S. Q.; Li, Y.; Yang, G. Q. Phys. Chem. Chem. Phys. 2011, 13, 2044.

[23] Tomalia, D. A.; Baker, H.; Dewald, J.; Hall, M. E.; Kallos, G.; Martin, S.; Roeck, J.; Ryder, J.; Smith, P. Polym. J. 1985, 17, 117. 